\title{
Asymptotic Analysis in Flow Curves for a Model of Soft Glassy Fluids
}

\author{
OLIVIER Julien*
}

May 14, 2009

\begin{abstract}
In this article we rigourously prove several asymptotical results for the flow curves of the Hébraud-Lequeux model, a rheological model which describes the behaviour of soft glassy fluids. This model has a control parameter $\alpha$ which governs the behaviour of the fluid at low shear rate. More precisely, we consider $\tau(\dot{\gamma})$ the stress in a block that is sheared at a constant rate $\dot{\gamma}$ and we prove that the system exhibits a transition in its behaviour at low shear rate when $\alpha$ goes through a critical value. The study is complicated by the fact that one of the parameter is only given implicitly and also we have to study two variables function in the neighbourhood of singularities.
\end{abstract}

Keywords: Non-newtonian fluids, Soft glassy rheology, Asymptotic analysis, Implicit functions

AMS Subject Classification: 35B40, 76A05

\section{Introduction}

In this article we are interested in a rheological model for glassy material in simple shear flow configuration. Glassy materials range from suspensions (like bentonite for example) to emulsions or to foams. Among other features, these materials exhibit a transition as a control parameter goes through a critical value. This control is for instance, in the case of suspensions, related to the density of solid particle in the fluid.

One model available to describe such material is the Hébraud-Lequeux (HL) model. In Section 2 we rapidly review the model and the physical quantities it involves. We prove in the following that this model exhibits such a transition.

Let us first give some notations. We note $H$ is the Heaviside function $\mathbf{1}_{\mathbf{R}^{+}}$ and $h(\sigma)=H(|\sigma|-1)$ (which is then a function that cuts off $\sigma$ in $[-1,1]$ ); we also note by $\delta_{0}$ the Dirac measure. The real constants $D e$ and $\lambda$ are given below while $b$ is a real parameter given by the experimental setting (it can thus vary but we can consider it a known data of the problem). The real constant $\mu$ is what we will call in the following the control parameter and, as $b$, may vary

*LAMA - UMR 5127 CNRS - Université de Savoie, UFR SFA, Campus scientifique, 73376 Le Bourget-du-Lac, Cedex, France 
but can always be considered given. Finally let us define $\Gamma(p)=\int_{|\sigma|>1} p(\sigma) \mathrm{d} \sigma$ a quantity sometimes called the fluidity. We are then looking for a density of probability $p$ that solves the following equation:

$$
-\lambda b \partial_{\sigma} p-\operatorname{Deh}(\sigma) p+\operatorname{De} \Gamma(p) \delta_{0}(\sigma)+\mu \operatorname{De} \Gamma(p) \partial_{\sigma}^{2} p=0 .
$$

This model thus presents a threshold (set to 1 in nondimensional form) and a relaxation to a zero stress state (described by the Dirac $\delta_{0}$ ) when the stress $\sigma$ grows beyond the threshold. The equation is supplemented with the following conditions:

$$
p \in \mathrm{H}^{1}(\mathbf{R}), p \quad \geq 0, \int_{\mathbf{R}} p(\sigma) \mathrm{d} \sigma=1 .
$$

Indeed $p$ represents the density of probability to find a block of material in stress $\sigma$. We treat here $b$ as a given independent variable (actually we shall rather use $y=\lambda b / D e$ as an independent variable) and keep it unconstrained. The variable $b$ is the nondimensional version of the shear rate $\dot{\gamma}$.

We state in Section 3 the main results of this paper whose Section 4, Section 5 and Section 6 serve as proof. In Section 4 we solve the differential equation (1) with conditions (2) that we see as a coupled system of a linear differential equation and a nonlinear implicit equation

$$
\begin{aligned}
0 & =-\lambda b \partial_{\sigma} p-\operatorname{Deh}(\sigma) p+\operatorname{De} \Gamma \delta_{0}(\sigma)+\mu D e \Gamma \partial_{\sigma}^{2} p \\
\Gamma & =\Gamma(p) .
\end{aligned}
$$

But what we are really interested in is the total stress in the material $\tau=$ $\int \sigma p \mathrm{~d} \sigma$ as a function of $\dot{\gamma}$ and, in particular, its behaviour at low shear that is to say when the shear rate $\dot{\gamma}$ tends to 0 . In order to obtain such behaviour we need to obtain first the behaviour as $y$ tends to 0 of the fluidity which is only given implicitly by (4) in Section 4.3. This behaviour depends on $\mu$, as we shall see in Section 5. We then turn to the main result of this paper, the asymptotic behaviour of $\tau$ in Section 6. Those two sections are fairly different since in 5 we try to compute the asymptotic of an implicitly defined function whereas in 6 we want to know if we can use this asymptotic to find the behaviour of a two variables function $\tau$ alongside a curve.

\section{Hébraud-Lequeux Model}

In this section we rapidly describe the rheological model that we use afterwards. To study a material at a continuous level we have to give a constitutive equation that links the stress with the deformation rate. For newtonian fluids this relation is simply expressing a linear relation between the stress and the deformation rate. For non newtonian (or complex) fluids there exists a wide variety of nonlinear laws. For example we have Bingham fluids or more generally HerschelBulkley fluids.

For the Hébraud-Lequeux (HL) model we go down to the mesoscopic scale, a scale where we can still consider the material continuous but where we can describe some heterogeneities in the material. The principle of this model is that a block of material at a mesoscopic level is not in a single stress state anymore but is described by a density of probability $p$ over the stress space. In 
a way, the block carries all possible stresses with a coefficient described by the density of probability $p$. The Hébraud-Lequeux equation describes the evolution of this density $p$ over time. In the following equation, $G_{0}$ and $T_{0}$ are physical constants, $\dot{\gamma}$ is the shear rate, and $\sigma_{c}$ is the relaxation threshold. We note $\Gamma(q)=\frac{1}{T_{0}} \int H\left(|\sigma|-\sigma_{c}\right) q(\sigma) \mathrm{d} \sigma$ and $D=\alpha \Gamma$ for a physical constant $\alpha$. We then have

$$
\left\{\begin{array}{l}
\partial_{t} p=-G_{0} \dot{\gamma} \partial_{\sigma} p+D(p) \partial_{\sigma}^{2} p-\frac{1}{T_{0}} H\left(|\sigma|-\sigma_{c}\right) p+\Gamma(p(t)) \delta_{0}(\sigma), \\
p(0, \sigma)=p_{0}(\sigma) .
\end{array}\right.
$$

The solution must also verify the following conditions:

$$
p \in \mathrm{L}_{t}^{2}\left(\mathrm{H}_{\sigma}^{1}(\mathbf{R})\right) \text { and } \quad \forall t p(t, .) \geq 0, \quad \int_{\mathbf{R}} p(t, \sigma) \mathrm{d} \sigma=1 .
$$

The quantity of interest for the physicist is the total stress or macroscopic stress since it is the one that can actually be measured in a rheometer ([7] for example). In the case of the HL model, the total stress $\tau$ at time $t$ is linked to the density of probability $p(t,$.$) via the formula \tau(p(t))=\int_{\sigma \in \mathbf{R}} \sigma p(t, \sigma) \mathrm{d} \sigma$.

We refer to HÉBRAud and LEqueux in [6] for details on the physical interpretation of this equation. What is important to note for this paper, is that the control parameter in the system is $\alpha$ and the critical value at which the transition takes place is $\sigma_{c}^{2} / 2$.

This equation has been studied from a mathematical point of view by É. CANCÈs, I. CATTO and Y. GATi in [2]. They have addressed the question of wellposedness of (5)-(6). For other mathematical studies on the time-dependent HL model we refer to the work of É. CAnCÈs, I. CATTO, Y. Gati and C. LE BRIS in [3] for the study of a micro-macro coupling of a Hébraud-Lequeux equation with a macroscopic velocity field which aims at describing the flow of a material in a Couette cell. These studies rely upon PDE methods. Another approach is a probabilistic approach via the theory of stochastic processes and martingales for which we refer to the work of M. BEN AlayA and B. Jourdain in [1].

É. CANCĖS ET AL. also looked at the stationary solutions of the equation, proving existence by giving the solution and its form (8) and stating uniqueness via the growth of a function. Since the uniqueness part was not so clear to us and because we work with different notations (some of the nondimensional constants that appear here where set to 1 in their paper) we give a proof of uniqueness in appendix A. The stationary solution solves an ODE in $\sigma$ which we obtain by formally removing the term $\partial_{t} p$ in (5). This is the starting point of our study. We shall actually work on the nondimensional version (1) which we give again below for convenience.

What we study in the following are the flow curves given by the model, that is to say the relation between a given shear rate $\dot{\gamma}$ and the corresponding macroscopic stress which is $\tau(p)$ with $p$ the stationary solution corresponding to $\dot{\gamma}$. The transition we observe is in the behaviour as $\dot{\gamma} \rightarrow 0$ of the flow curve.

Let us now explain how we obtain the nondimensional system (1) we use in the rest of this work. We take the same notations as Y. GATI in her PHD thesis [5]. We have to choose a time scale $T$ which is the duration of the physical experimentation (thus the material may not have the same properties if you 
observe it for a long or a short time); the stress scale is given by $\sigma_{c}$ then all of the quantities appearing in (5) become:

$$
t^{\prime}=t / T, \sigma^{\prime}=\sigma / \sigma_{c}, b=T \dot{\gamma}
$$

and

$$
p^{\prime}\left(t^{\prime}, \sigma^{\prime}\right)=\sigma_{c} p\left(T t^{\prime}, \sigma_{c} \sigma^{\prime}\right), \quad \Gamma^{\prime}\left(p^{\prime}\left(t^{\prime}\right)\right)=\int_{\left|\sigma^{\prime}\right|>1} p^{\prime}\left(t^{\prime}, \sigma^{\prime}\right) \mathrm{d} \sigma^{\prime}=T_{0} \Gamma\left(p\left(T t^{\prime}\right)\right) .
$$

and the following nondimensional constants appear in the system

$$
D e=T / T_{0} \quad \mu=\alpha / \sigma_{c}^{2} \quad \lambda=G_{0} / \sigma_{c}
$$

We find here $D e$ the so-called Deborah number.

Then the nondimensional stationary Hébraud-Lequeux equation we have obtained is (we have dropped the primes for the sake of clarity):

$$
-\lambda b \partial_{\sigma} p-\operatorname{Deh}(\sigma) p+\operatorname{De} \Gamma(p) \delta_{0}(\sigma)+\mu \operatorname{De} \Gamma(p) \partial_{\sigma}^{2} p=0 .
$$

The conditions on $p$ read the same as in the time-dependent case, namely:

$$
p \in \mathrm{H}^{1}(\mathbf{R}), \quad p \geq 0, \quad \int_{\mathbf{R}} p(\sigma) \mathrm{d} \sigma=1 .
$$

A related topic to the study of stationary solutions of the HL model is the question of long-time behaviour of the solution to the time-dependent equation. This has been addressed in some cases by É. CANCÈs and C. LE BRIS in [4] by an entropy-decay method.

\section{Presentation of the Main Results}

For the sake of self-containment, before we state the main result of this paper we give again the existence and uniqueness theorem we rely upon:

Proposition 1. For all given $b>0$ there exists a unique solution $p(b)$ to equation (1) with conditions (2).

This proposition is stated in more detail (with the actual form of $p(b)$ ) and proved in Section 4 (See also Appendix A for details on the uniqueness part which requires a special care). As a byproduct of the proof of this theorem we have that there exists a function $\phi_{\mu}$ such that $\Gamma(p(b))=\int_{|\sigma|>1} p(b, \sigma) \mathrm{d} \sigma=$ $\phi_{\mu}(\lambda b / D e) / \mu$ with the following behaviour:

Corollary 1. For every $\mu$ there exists a non negative constant $c(\mu)$ such that, as y decreases to 0 , we have:

if $\mu>1 / 2, \phi_{\mu}(y) \rightarrow c(\mu)$,

if $\mu<1 / 2, \phi_{\mu}(y) \sim c(\mu) y$,

if $\mu=1 / 2, \phi_{1 / 2}(y) \sim c(1 / 2) y^{4 / 5}$. 


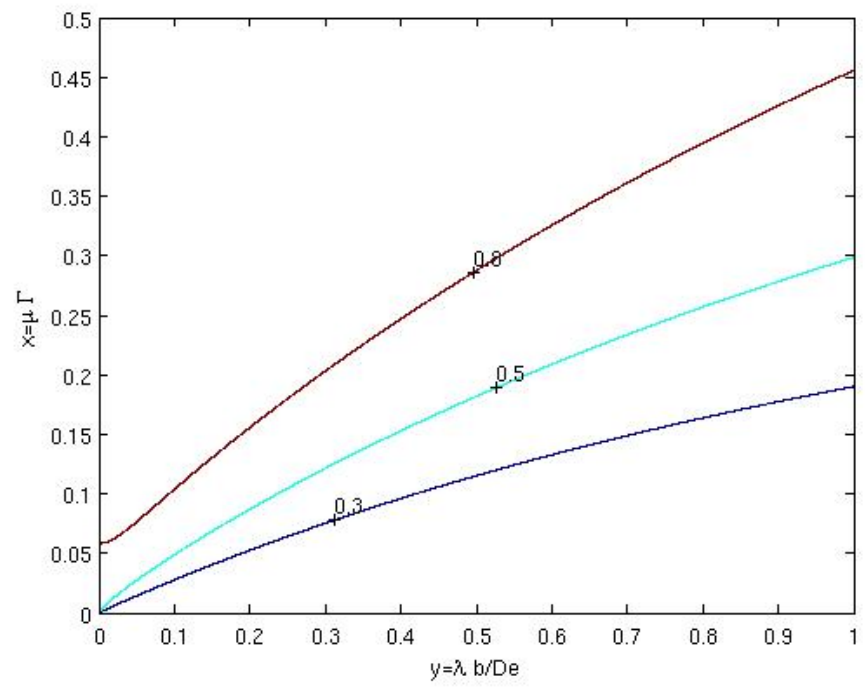

Figure 1: Numerical Representation of $\phi_{\mu}$ for $\mu=0.3,0.5$ and 0.8 .

These behaviours can be seen on Figure 1. The definition of $c(\mu)$ in each case is given alongside the proof of this corollary in Section 5. We can now state (in physical variables) the main result of this paper:

Theorem 1. We note $\eta_{c}=G_{0} T_{0}$. Let $p(\dot{\gamma}, \sigma)$ be the unique solution given by Proposition 1 at shear rate $\dot{\gamma}$. Then we have the following behaviours for the function $\tau(\dot{\gamma})=\int_{\sigma \in \mathbf{R}} \sigma p(\dot{\gamma}, \sigma) \mathrm{d} \sigma$ at $\dot{\gamma} \rightarrow 0$ :

If $\alpha>\sigma_{c}^{2} / 2$ the fluid behaves like a newtonian fluid with an apparent viscosity $\eta\left(\alpha / \sigma_{c}^{2}\right)$. Let us first define $\eta^{*}(\mu)$ via the formula

$$
\eta^{*}(\mu)=1+\frac{3 \mu-1+1 / 2(4 \mu-1)^{3 / 2}}{24 \mu(\mu-1 / 2)^{2}}
$$

We then have

$$
\tau(\dot{\gamma}) \sim \eta\left(\alpha / \sigma_{c}^{2}\right) \dot{\gamma}
$$

where the apparent viscosity $\eta$ is given by $\eta\left(\alpha / \sigma_{c}^{2}\right)=\eta^{*}\left(\alpha / \sigma_{c}^{2}\right) \eta_{c}$.

If $\alpha<\sigma_{c}^{2} / 2$ the fluid presents a stress threshold $\tau_{0}$. We first define $c$ to be the unique positive root of the following equation:

$$
\frac{c(\cosh (1 / c)-1)}{\sinh (1 / c)}=\frac{\alpha}{\sigma_{c}^{2}}
$$

and $\tau^{*}$ by the formula

$$
\tau^{*}=\left(\frac{\alpha}{2 \sigma_{c}^{2}}-1\right) c
$$

We the have

$$
\tau(\dot{\gamma}) \rightarrow \tau_{0}\left(\alpha / \sigma_{c}^{2}\right)
$$

with $\tau_{0}$ given by $\tau_{0}\left(\alpha / \sigma_{c}^{2}\right)=\tau^{*}\left(\alpha / \sigma_{c}^{2}\right) \sigma_{c}$. 


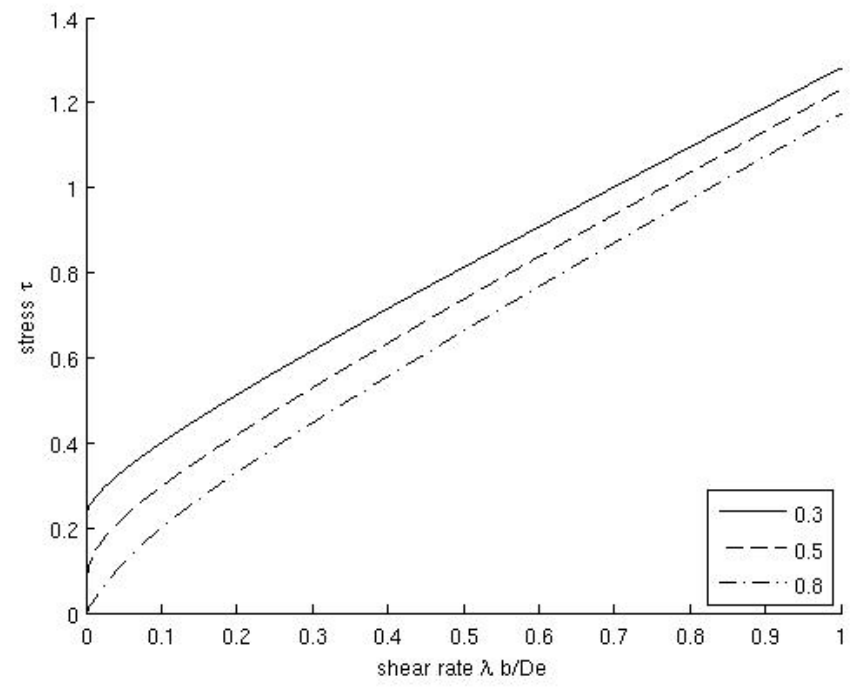

Figure 2: Numerical Representation of $\tau$ for $\mu=0.3,0.5$ and 0.8 .

If $\alpha=\sigma_{c}^{2} / 2$ the fluid follows a power law with exponent $1 / 5$ meaning that

$$
\tau(\dot{\gamma}) \sim \frac{24^{2 / 5}}{12} \sigma_{c}^{4 / 5} \eta_{c}^{1 / 5} \dot{\gamma}^{1 / 5}
$$

This theorem is important because it describes a transition that is one of the characteristic features of glassy materials. Figure 2 shows the different kind of behaviour one may find. These asymptotic results can be found without proof in Hébraud's PHD thesis and subsequently on several other Physics paper. But this is actually the first time that a mathematical proof of this theorem is given. We were also able to give the numerical constants in the asymptotic for all $\mu$ whereas only the cases $\mu \rightarrow 1 / 2$ had been given. The following corollary might be useful too:

Corollary 2. When $\mu=\alpha / \sigma_{c}^{2}$ is close to $1 / 2$ we have:

if $\mu>1 / 2, \eta^{*}(\mu) \sim \frac{1}{12}\left(\mu-\frac{1}{2}\right)^{-2}$

if $\mu<1 / 2, \tau^{*}(\mu) \sim \frac{1}{\sqrt{6}}\left(\frac{1}{2}-\mu\right)^{1 / 2}$

\section{Construction of the Solution}

In this section we want to prove the following proposition (which is only a more detailed restatement of Proposition 1):

Proposition 2. There exists a unique solution p to equation (1) with conditions 
(2) which is of the form:

$$
p(\sigma, b)= \begin{cases}\alpha_{+} \exp \left(\beta_{+}(\sigma+1)\right) & \text { if } \sigma \leq-1 \\ \alpha_{+}\left(1-\rho \beta_{+}+\rho \beta_{+} \exp ((\sigma+1) / \rho)\right) & \text { if }-1<\sigma \leq 0 \\ \alpha_{-}\left(1-\rho \beta_{-}+\rho \beta_{-} \exp ((\sigma-1) / \rho)\right) & \text { if } 0<\sigma \leq 1 \\ \alpha_{-} \exp \left(\beta_{-}(\sigma-1)\right) & \text { if } 1<\sigma\end{cases}
$$

where $\alpha_{+}$and $\alpha_{-}$depend on $b$ and a variable $\Gamma$. There also exists a function $g$ such that we have $g(\mu \Gamma, \lambda b / D e)=\mu$ (we give it in section 4.3). Actually we have $\Gamma=\Gamma(p)$. Moreover, since $\partial_{x} g(x, y)>0$ for all $x$ and $y$ non negative then there exists a function $\phi_{\mu}$ globally defined that gives $\Gamma=\phi_{\mu}(\lambda b / D e) / \mu$.

The constants $\rho, \beta_{+}, \beta_{-}$will be defined in the following in terms of the physical constants.

To prove this proposition we will in fact, only expand the computations of [2], in our own framework (mainly we have different notations) only for the sake of self-containment. The idea is pretty simple. First of all, the nonlinear differential equation is decoupled as a linear differential equation and a nonlinear consistency equation. If not for the Dirac measure (which is zero almost everywhere anyway) the linear differential equation could be easily solved by means of the characteristic polynomial of the equation. We thus treat here the Dirac as zero and solve the equation on $\mathbf{R}^{*}$. This will leave us three degree of freedom (but one will be taken care of by the consistency equation) and we fix the other two by imposing the constraint on regularity and integral. Finally we verify that the function we construct is a solution to the initial problem.

We work under the assumption that $b$ is non negative. The equation (1) is nonlinear because of the coefficient $\Gamma(p)$. To alleviate this difficulty we simply rewrite (1) as (3)-(4)

$$
\begin{aligned}
& -\lambda b \partial_{\sigma} p-\operatorname{Deh}(\sigma) p+D e \Gamma \delta_{0}(\sigma)+\mu D e \Gamma \partial_{\sigma}^{2} p=0 \\
& \Gamma=\Gamma(p)
\end{aligned}
$$

To proceed further we attack the first equation considering that $\Gamma$ is a given non negative constant and we afterwards fix $\Gamma$ using the second equation of the above system.

\subsection{Solving the linear equation in $\mathcal{D}^{\prime}\left(\mathbf{R}^{*}\right)$}

We solve (3) first in $\mathcal{D}^{\prime}\left(\mathbf{R}^{*}\right)$ which amounts to say that we turn the Dirac to 0 namely we are looking at the equation:

$$
0=-\lambda b \partial_{\sigma} p-\operatorname{Deh}(\sigma) p+\mu D e \Gamma \partial_{\sigma}^{2} p
$$

on $\mathbf{R}^{*}$. It can easily be solved on each open interval $]-\infty,-1[]-1,,0[] 0,,1[$ and ] $1, \infty[$ and since the equation is of order two on each interval, we have two degrees of freedom to tune the solution on each interval. On the unbounded interval we set one of the constant to 0 in order to suppress an exponential growth that would prevent the solution from being in $\mathrm{L}^{1}$. Next we fix the constant on the other interval by enforcing $\mathcal{C}^{1}$ regularity at -1 and 1 . Now one could wonder why $\mathcal{C}^{1}$ while we are looking for solution in $\mathrm{H}^{1}(\mathbf{R})$ which are merely continuous 
on the real line. It is because if there were jumps in the derivative of $p$ at -1 or 1, this would induce Dirac measures at these points in the second order derivative and we have nothing in the equation that would compensate these Dirac measures.

Expression of the Solution of (3). The functions $p$ which solve (3) in the sense of distribution on $\mathbf{R}^{*}$ and which are in $\mathcal{C}^{1}\left(\mathbf{R}^{*}\right)$ and verify

$$
\int_{\mathbf{R}^{*}} p^{2}+\int_{\mathbf{R}^{*}}\left(\partial_{\sigma} p\right)^{2}<\infty
$$

are given by the following formulae:

$$
p(\sigma)= \begin{cases}\alpha_{+} \exp \left(\beta_{+}(\sigma+1)\right) & \text { if } \sigma \leq-1 \\ \alpha_{+}\left(1-\rho \beta_{+}+\rho \beta_{+} \exp \left(\frac{1}{\rho}(\sigma+1)\right)\right) & \text { if }-1<\sigma \leq 0 \\ \alpha_{-}\left(1-\rho \beta_{-}+\rho \beta_{-} \exp \left(\frac{1}{\rho}(\sigma-1)\right)\right) & \text { if } 0<\sigma \leq 1 \\ \alpha_{-} \exp \left(\beta_{-}(\sigma-1)\right) & \text { if } 1<\sigma\end{cases}
$$

where $\beta_{+}, \beta_{-}$are the roots (respectively non negative and non positive) of the equation

$$
\mu D e \Gamma X^{2}-\lambda b X-D e=0
$$

and $\rho$ is $\mu \operatorname{De} \Gamma /(\lambda b)=1 /\left(\beta_{+}+\beta_{-}\right)$. By the definition of $\beta_{ \pm}$and $\rho$ we remark that we have:

$$
1-\rho \beta \pm=\rho \beta \mp .
$$

The degrees of freedom are now $\alpha_{+}$and $\alpha_{-}$.

Determination of the $\alpha_{ \pm}$used in (8). To fix $\alpha_{+}$and $\alpha_{-}$we impose continuity at $\sigma=0$ (because as we said, we are interested in solutions in $\mathrm{H}^{1}(\mathbf{R})$ thus continuous) and $\int p=1$. These two conditions give us the following system on $\alpha_{+}, \alpha_{-}$:

$$
\left\{\begin{aligned}
0= & \left(1-\rho \beta_{+}+\rho \beta_{+} e^{1 / \rho}\right) \alpha_{+}-\left(1-\rho \beta_{-}+\rho \beta_{-} e^{-1 / \rho}\right) \alpha_{-} \\
1= & \left(\frac{1}{\beta_{+}}+\left(1-\rho \beta_{+}\right)+\rho^{2} \beta_{+}\left(e^{1 / \rho}-1\right)\right) \alpha_{+} \\
& +\left(\frac{-1}{\beta_{-}}+\left(1-\rho \beta_{-}\right)+\rho^{2} \beta_{-}\left(1-e^{-1 / \rho}\right)\right) \alpha_{-}
\end{aligned}\right.
$$

Existence of $\alpha_{ \pm}$. Provided that the determinant of this system is not zero we have fixed a unique couple $\left(\alpha_{+}, \alpha_{-}\right)$. Let us check that this is the case by computing this determinant:

$$
\begin{aligned}
\Delta= & 2-\rho^{2} \beta_{+} \beta_{-}\left(\cosh \left(\frac{1}{\rho}\right)-1\right)+\cosh \left(\frac{1}{\rho}\right)+\left(-\frac{1}{\rho \beta_{+} \beta_{-}}+3 \rho\right) \sinh \left(\frac{1}{\rho}\right) \\
& +\left(\beta_{+}-\beta_{-}\right)\left[\rho^{2}\left(\cosh \left(\frac{1}{\rho}\right)-1\right)+\rho \sinh \left(\frac{1}{\rho}\right)-\frac{1}{\beta_{+} \beta_{-}} \cosh \left(\frac{1}{\rho}\right)\right] .
\end{aligned}
$$

We see that in fact $\Delta>0$ (remember that $\beta_{+}>0, \beta_{-}<0$ and since $b>0$, $1 / \rho>0)$. 
Positivity of $\alpha_{ \pm}$. At this point let us also check that $\alpha_{ \pm}>0$. We have by Cramer's rule for linear systems:

$$
\Delta \alpha_{+}=1-\rho \beta_{-}+\rho \beta_{-} e^{-1 / \rho} \text { and } \Delta \alpha_{-}=1-\rho \beta_{+}+\rho \beta_{+} e^{1 / \rho} .
$$

Using Remark (11), we have $1-\rho \beta_{-}+\rho \beta_{-} e^{-1 / \rho}$ equal to $\rho\left(\beta_{+}+\beta_{-} e^{-1 / \rho}\right)$. But since $\beta_{-}<0$ and $\rho>0$ we have $e^{-1 / \rho}<1$ and $\beta_{-} e^{-1 / \rho}>\beta_{-}$and thus $\beta_{+}+\beta_{-} e^{-1 / \rho}>\beta_{+}+\beta_{-}>0$. Consequently, $\alpha_{+}>0$ and the same holds true for $\alpha_{-}$. Returning to equation (8) we see that the positivity of $\alpha_{ \pm}$gives the positivity of the function $p$ on $]-\infty,-1[$ and $] 1,+\infty\left[\right.$. But since $\alpha_{+}, \beta_{+}$and $\rho$ are non negative, it turns out that $p$ is increasing on the interval ] - 1,0[ and since its value at -1 is by construction $\alpha_{+}>0, p$ remains non negative on $]-1,0[$. Again the same holds true on $] 0,1\left[\right.$. Note that from (13)-(14), $\alpha_{ \pm}$ depend on $\rho$ and $\beta_{ \pm}$which in turn depend on $b$ and $\Gamma$.

\subsection{Solving the nonlinear problem (3)-(4)}

Fixing $\Gamma$ using (4). Let us sum up what we have obtained so far. We have now a function $p$ which lies in $\mathrm{H}^{1}(\mathbf{R})$ that solve $(3)$ in the space $\mathcal{D}^{\prime}\left(\mathbf{R}^{*}\right)$ that is to say in the sense of distribution but with test functions identically zero in a neighbourhood of zero. We also have that $p>0$ and $\int p=1$, the second constraint being exactly the second equation of system (12) and we have so, for every non negative $\Gamma$. We now adjust $\Gamma$, so that (4) is satisfied. To do this we integrate $p$ for $|\sigma|>1$ using (8). In terms of $\alpha_{ \pm}$, (4) amounts to:

$$
\frac{\alpha_{+}}{\beta_{+}}-\frac{\alpha_{-}}{\beta_{-}}=\Gamma \text {. }
$$

This is a nonlinear implicit equation on $\Gamma$ because as we mentionned before $\alpha_{ \pm}$ and $\beta_{ \pm}$depend on $\Gamma$. Let us admit for the moment that this equation has a unique solution which means that as long as we give a non negative $b$ we can find a unique non negative $\Gamma$ that satisfies (15). Let us see now that we have found a solution to (1).

Proof that $p$ solves $(1)$ in $\mathcal{D}^{\prime}(\mathbf{R})$. We now show that $p$ verifies in fact (1) on the whole real line. We note that $p$ is continous on the real line and differentiable except at $\sigma=0$. Its derivative in $\mathcal{D}^{\prime}(\mathbf{R})$ is thus its derivative almost everywhere. The derivative $\partial_{\sigma} p$ being continous except at $\sigma=0$ and differentiable except at $\sigma \in\{0,-1,1\}$ its derivative is its derivative almost everywhere plus the jump at $0,\left[\partial_{\sigma} p\right]_{0} \delta_{0}(\sigma)$. Since $p$ verifies (1) almost everywhere, it is only left to show that:

$$
\begin{aligned}
-\mu D e \Gamma\left[\partial_{\sigma} p\right]_{0}+D e \Gamma & =\mu D e \Gamma\left(\alpha_{-} \beta_{-} e^{-1 / \rho}-\alpha_{+} \beta_{+} e^{1 / \rho}\right)+D e \Gamma \\
& =0
\end{aligned}
$$

which becomes

$$
\alpha_{+} \beta_{+} e^{1 / \rho}-\alpha_{-} \beta_{-} e^{-1 / \rho}=1 / \mu .
$$

We multiply (16) by $\Delta$ and use (14) to obtain:

$$
\beta_{+} e^{1 / \rho}\left(1-\rho \beta_{-}+\rho \beta_{-} e^{-1 / \rho}\right)-\beta_{-} e^{-1 / \rho}\left(1-\rho \beta_{+}+\rho \beta_{+} e^{1 / \rho}\right)=\Delta / \mu
$$


which is equivalent to

$$
\beta_{+} e^{1 / \rho}\left(1-\rho \beta_{-}\right)-\beta_{-} e^{-1 / \rho}\left(1-\rho \beta_{+}\right)=\Delta / \mu
$$

and then to

$$
\rho\left(\beta_{+}^{2} e^{1 / \rho}-\beta_{-}^{2} e^{-1 / \rho}\right)=\Delta / \mu .
$$

That's what we want to prove.

We now use (15) to obtain:

$$
\frac{\alpha_{+}}{\beta_{+}}-\frac{\alpha_{-}}{\beta_{-}}=\Gamma \Leftrightarrow \alpha_{+} \beta_{-}-\alpha_{-} \beta_{+}=\Gamma \beta_{+} \beta_{-} .
$$

Recalling that $\beta_{ \pm}$are roots of equation (10), we have $\beta_{+} \beta_{-}=-1 / \mu \Gamma$ and therefore $\alpha_{+} \beta_{-}-\alpha_{-} \beta_{+}=-1 / \mu$. We multiply by $-\Delta$ this equality and use (14) to obtain:

$$
\begin{aligned}
\frac{\Delta}{\mu} & =\beta_{+}\left(\Delta \alpha_{-}\right)-\beta_{-}\left(\Delta \alpha_{+}\right) \\
& =\beta_{+}\left(1-\rho \beta_{+}+\rho \beta_{+} e^{1 / \rho}\right)-\beta_{-}\left(1-\rho \beta_{-}+\rho \beta_{-} e^{-1 / \rho}\right) \\
& =\beta_{+}\left(\rho \beta_{-}+\rho \beta_{+} e^{1 / \rho}\right)-\beta_{-}\left(\rho \beta_{+}+\rho \beta_{-} e^{-1 / \rho}\right) \\
& =\rho\left(\beta_{+}^{2} e^{1 / \rho}-\beta_{-}^{2} e^{-1 / \rho}\right) .
\end{aligned}
$$

The jump condition is thus verified. We can see in fact that (16) is equivalent to (15). Thus the only solution in $\mathcal{D}(\mathbf{R})$ of (3) are in fact solutions of (1). This is not that surprising since if one integrates equation (3) over the real line you obtain a priori that $\Gamma=\Gamma(p)$.

What is left now is to understand how we can use (15) to fix $\Gamma$ and be sure that, as we claimed, there exists a unique $\Gamma$ that fits the consistency equation.

\subsection{Implicit relations that link $\Gamma$ and $b$}

To obtain an explicit function that gives implicitly $\Gamma$ we just have to use (15) and use the value of $\alpha_{ \pm}$given by (13) and (14) . It is easier to use new variables defined by $x=\mu \Gamma$ and $y=\lambda b / D e$. Actually $x$ is the nondimensional diffusion coefficient in stress space while $y=\dot{\gamma} / \dot{\gamma}_{c}$ with $\dot{\gamma}_{c}=G_{0} T_{0} / \sigma_{c}$ the critical shear rate in the system. Let us begin by rewriting $\Delta$ with these variables:

$$
\begin{aligned}
& \Delta(x, y)=\frac{2 x}{y^{2}}\left(\cosh \left(\frac{y}{x}\right)-1\right)+\cosh \left(\frac{y}{x}\right)+\left(\frac{3 x}{y}+y\right) \sinh \left(\frac{y}{x}\right) \\
& +\left(\left(\frac{y}{x}\right)^{2}+\frac{4}{x}\right)^{1 / 2}\left[\left(\frac{x}{y}\right)^{2}\left(\cosh \left(\frac{y}{x}\right)-1\right)+\frac{x}{y} \sinh \left(\frac{y}{x}\right)+x \cosh \left(\frac{y}{x}\right)\right]
\end{aligned}
$$

Now using the consistency equation (15) we find that

$$
\Delta \Gamma=\left(2 \rho-1 /\left(\rho \beta_{+} \beta_{-}\right)\right) \sinh (1 / \rho)-\left(\beta_{+}-\beta_{-}\right) /\left(\beta_{+} \beta_{-}\right) \cosh (1 / \rho)
$$


which we write in the $(x, y)$ variables using the actual values of $\beta_{ \pm}$in terms of the coefficient of (10)

$$
\frac{1}{\mu} \Delta(x, y) x=x\left(\left(\frac{y}{x}\right)^{2}+\frac{4}{x}\right)^{1 / 2} \cosh \left(\frac{y}{x}\right)+\left(2 \frac{x}{y}+y\right) \sinh \left(\frac{y}{x}\right) .
$$

We now have the desired implicit function linking $\Gamma$ and $b$ :

$$
\begin{aligned}
f_{\mu}(x, y)= & \frac{1}{\mu}\left[\frac{2 x^{2}}{y^{2}}\left(\cosh \left(\frac{y}{x}\right)-1\right)+x \cosh \left(\frac{y}{x}\right)+\left(\frac{3 x^{2}}{y}+x y\right) \sinh \left(\frac{y}{x}\right)\right. \\
& \left.+\sqrt{y^{2}+4 x}\left(\frac{x^{2}}{y^{2}}\left(\cosh \left(\frac{y}{x}\right)-1\right)+\frac{x}{y} \sinh \left(\frac{y}{x}\right)+x \cosh \left(\frac{y}{x}\right)\right)\right] \\
& -\sqrt{y^{2}+4 x} \cosh \left(\frac{y}{x}\right)-\left(\frac{2 x}{y}+y\right) \sinh \left(\frac{y}{x}\right) .
\end{aligned}
$$

and we want $f_{\mu}(x, y)=0$. Interestingly enough, this function depends on the nondimensional control parameter.

On the open quarter plane $\{x>0, y>0\}, f_{\mu}(x, y)=0$ is equivalent to $g(x, y)=\mu$ with:

$$
\begin{aligned}
g(x, y)=\frac{\frac{2 x^{2}}{y^{2}}\left(\cosh \left(\frac{y}{x}\right)-1\right)+x \cosh \left(\frac{y}{x}\right)+\left(\frac{3 x^{2}}{y}+x y\right) \sinh \left(\frac{y}{x}\right)}{\sqrt{y^{2}+4 x} \cosh \left(\frac{y}{x}\right)+\left(\frac{2 x}{y}+y\right) \sinh \left(\frac{y}{x}\right)} \\
\frac{+\sqrt{y^{2}+4 x}\left(\frac{x^{2}}{y^{2}}\left(\cosh \left(\frac{y}{x}\right)-1\right)+\frac{x}{y} \sinh \left(\frac{y}{x}\right)+x \cosh \left(\frac{y}{x}\right)\right)}{\sqrt{y^{2}+4 x} \cosh \left(\frac{y}{x}\right)+\left(\frac{2 x}{y}+y\right) \sinh \left(\frac{y}{x}\right)} .
\end{aligned}
$$

It is easy to check that for a given $y>0$ the function $x \mapsto g(x, y)$ tends to 0 as $x$ tends to 0 and tends to infinity as $x$ tends to infinity. Since it is obviously continuous, this guarantees that there exists at least one $\Gamma$ that solves the consistency equation and we have at least one solution to our problem. In fact we can show that $\partial_{x} g(x, y)>0$ for all $x$ and $y$ non negative. This gives us at the same time the uniqueness we claimed and the regularity of the function giving $x$ in terms of $y$. We now introduce $\phi_{\mu}$ the implicit function satisfying

$$
\forall y>0 \forall \mu>0 \quad f_{\mu}(x, y)=0 \Leftrightarrow \quad g(x, y)=\mu \Leftrightarrow \quad x=\phi_{\mu}(y) .
$$

We refer to Appendix A for some details on the computations that are needed to show $\partial_{x} g(x, y)>0$.

\section{$5 \quad$ Asymptotic Behaviour of $\phi_{\mu}(y)$ at $y \rightarrow 0$}

As we have seen, it is easier to think of the solution to (1) as depending of two parameters $x$ and $y$ and when looking for $y$ dependent behaviour to remember that $x$ and $y$ are linked via $x=\phi_{\mu}(y)$. Before we can turn to the main point of 
this paper, that is to say the behaviour of the macroscopic stress as $y$ decreases to 0 , we will need the behaviour of $\phi_{\mu}$ as $y$ decreases to 0 explicitly. The function $\phi_{\mu}$ has different behaviours with different values of $\mu$ which is the first sign of the transition. We want in this section to prove that:

Proposition 3. For every $\mu$ we introduce $\phi_{\mu}^{\mathrm{app}}$, the first non zero term of the expansion of $\phi_{\mu}$ at $y=0$ that is to say we have $\phi_{\mu}(y) \sim \phi_{\mu}^{\mathrm{app}}(y)$. Then there exists a non negative constant $c(\mu)$ such that when $y$ decreases to 0 we have:

if $\mu>1 / 2, \phi_{\mu}^{\operatorname{app}}(y)=c(\mu)$,

if $\mu<1 / 2, \phi_{\mu}^{\operatorname{app}}(y)=c(\mu) y$,

if $\mu=1 / 2, \phi_{\mu}^{\mathrm{app}}(y)=c(1 / 2) y^{4 / 5}$.

We now turn to the proof of this proposition

\subsection{The simplest case $\mu>1 / 2$}

The function $f_{\mu}$ constructed via (18) has a very wild behaviour at the neigbourhood of $(0,0)$. However, it can be continued (with the same formula) with a $\mathcal{C}^{\infty}$ regularity on the domain $\mathbf{R}_{+}^{*} \times \mathbf{R}$. We then have:

$$
f_{\mu}(c, 0)=\frac{1+3 \sqrt{c}+4 c+2 c \sqrt{c}}{\mu}-2 \sqrt{c}-2
$$

for any non negative $c$. The function $\zeta: c \mapsto(1+3 \sqrt{c}+4 c+2 c \sqrt{c}) /(2 \sqrt{c}+2)$, is strictly increasing over $\mathbf{R}_{+}$and tends to $1 / 2$ as $c \rightarrow 0$. Consequently, for every $\mu>1 / 2$ there exists $c(\mu)>0$ such that $\zeta(c(\mu))=\mu$ which amounts to $f_{\mu}(c(\mu), 0)=0$. As a matter of fact, one can excplicitly compute

$$
c(\mu)=\mu-\frac{\sqrt{4 \mu-1}}{2} .
$$

On the other side, $\partial_{x} f_{\mu}(c(\mu), 0)$ cannot be zero. We can then apply the implicit function theorem for $f_{\mu}$ at point $(c(\mu), 0)$ and with the uniqueness property we have made for $\phi_{\mu}(y)$ we have that $\forall \mu>1 / 2, \lim _{y \rightarrow 0} \phi_{\mu}(y)=c(\mu)$. For $\mu>1 / 2$ we pose $\phi_{\mu}^{\text {app }}(y)=c(\mu)$.

As a matter of fact we will need another term in the expansion of $\phi_{\mu}$ at $y=0$ which we can obtain in this case by computing the derivatives of $\phi_{\mu}$. We find that we have $\phi_{\mu}^{\prime}(0)=0$ and $\phi_{\mu}^{\prime \prime}(0) \neq 0$. Thus we have

$$
\phi_{\mu}(y)-\phi_{\mu}^{\mathrm{app}}(y) \sim c_{1}(\mu) y^{2}
$$

as $y$ goes to zero.

\subsection{Preliminary computation for cases $\mu \leq 1 / 2$}

We can note that as $\mu$ tends to $1 / 2, c(\mu)$ tends to 0 , leading us to believe that as $\mu$ tends to $1 / 2, \phi_{\mu}(y)$ tends to 0 as $y$ tends to 0 . We thus need to make a change of variable in order to alleviate the difficulty in the vicinity of $(0,0)$. We then pose $X=x$ and $Y=y / x$. The map $T:(x, y) \mapsto(x, y / x)$ is a $\mathcal{C}^{1}$-diffeomorphism of $\mathbf{R}_{+}^{*} \times \mathbf{R}$ in itself whose inverse map is $T^{-1}:(X, Y) \mapsto(X, Y X)$.

We then get a new function: 


$$
\begin{aligned}
F_{\mu}(X, Y)= & \left(f_{\mu} \circ T^{-1}\right)(X, Y) \\
= & \frac{1}{\mu}\left[\frac{2(\cosh (Y)-1)}{Y^{2}}+X \cosh (Y)+\left(3 \frac{X}{Y}+X^{2} Y\right) \sinh (Y)\right] \\
& -\left(\frac{2}{Y}+Y X\right) \sinh (Y) \\
+ & \sqrt{Y^{2} X^{2}+4 X} \times \\
& \left(\frac{1}{\mu}\left[\frac{\cosh (Y)-1}{Y^{2}}+\frac{\sinh (Y)}{Y}+X \cosh (Y)\right]-\cosh (Y)\right) .
\end{aligned}
$$

Let us note that this function does not have a singularity at $Y=0$. In the $\mu<1 / 2$ case we wish to show that $(X, Y)=T\left(\phi_{\mu}(y), y\right)$ tends to $(0,1 / c(\mu))$ with $c(\mu)>0$ and in the $\mu=1 / 2$ case we wish to show that $(X, Y)$ tends to $(0,0)$. We thus need to study $F_{\mu}$ in a neighborhood of the axis $X=0$. Indeed, $F_{\mu}$ can be continuously continued at $\{X=0\}$. However, due to the square root, the derivatives of $F_{\mu}$ tends to $+\infty$ as $X$ tends to 0 . It is, thus, not possible to continue $F_{\mu}$ with a $\mathcal{C}^{1}$ regularity on a neighbourhood of the axis $\{X=0\}$ and we will not be able to apply the implicit function theorem directly on $F_{\mu}$ as we $\operatorname{did}$ on $f_{\mu}$ for $\mu>1 / 2$.

We remark that if we note

$$
\begin{aligned}
& A_{\mu}(X, Y)=\frac{1}{\mu}\left[\frac{2(\cosh (Y)-1)}{Y^{2}}+X \cosh (Y)+\left(3 \frac{X}{Y}+X^{2} Y\right) \sinh (Y)\right] \\
&-\left(\frac{2}{Y}+Y X\right) \sinh (Y) \\
& B(X, Y)= Y^{2} X^{2}+4 X \\
& C_{\mu}(X, Y)= \frac{1}{\mu}\left[\frac{\cosh (Y)-1}{Y^{2}}+\frac{\sinh (Y)}{Y}+X \cosh (Y)\right]-\cosh (Y)
\end{aligned}
$$

$F_{\mu}$ is in reality of the form

$$
F_{\mu}(X, Y)=A_{\mu}(X, Y)+\sqrt{B(X, Y)} C_{\mu}(X, Y) .
$$

We want to remove the square root to remove the problem of the continuity of the derivative. We thus introduce the function $G_{\mu}$ defined by $G_{\mu}(X, Y)=$ $A_{\mu}(X, Y)^{2}-B(X, Y) C_{\mu}(X, Y)^{2}$. The relation $F_{\mu}(X, Y)=0$ clearly implies $G_{\mu}(X, Y)=0$. But we are interested in the reverse implication and pay attention to the signs of the terms in the equation. We have in fact that $F_{\mu}(X, Y)=0$ is equivalent to: 


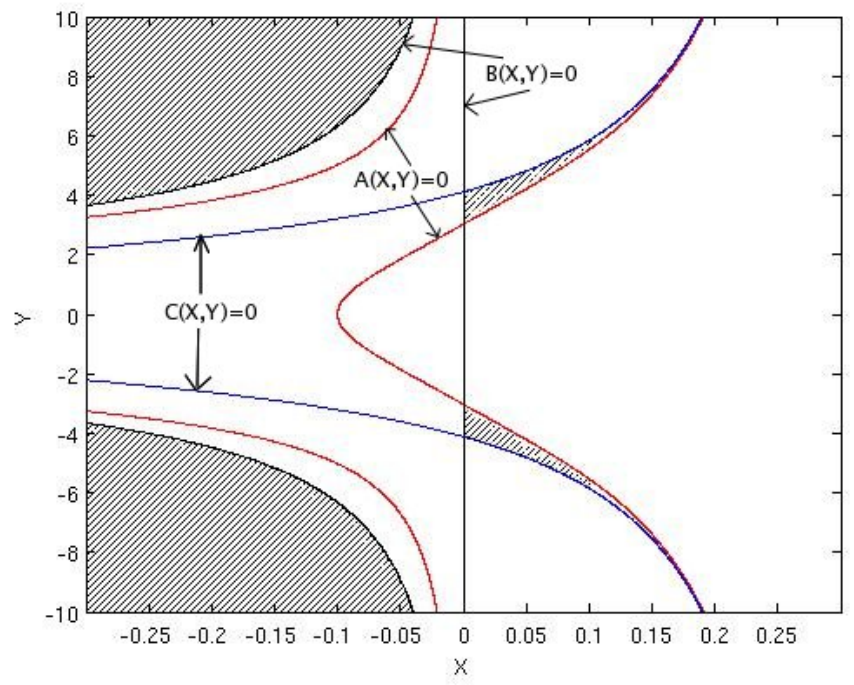

Figure 3: Setting of the $A, B$ and $C$ functions in the $(X, Y)$ plane for $\mu=0.3$. The grey area corresponds to the parts where $F_{\mu}(X, Y)=0 \Leftrightarrow G_{\mu}(X, Y)=0$. The curves are defined on the whole plane even if only the part $X>0$ is physically relevant.

$$
\begin{aligned}
& \left\{\begin{array}{l}
B(X, Y)=0 \\
A_{\mu}(X, Y)=0
\end{array}\right. \\
& \text { or }\left\{\begin{array}{l}
B(X, Y)>0 \\
A_{\mu}(X, Y)=0 \\
C_{\mu}(X, Y)=0
\end{array}\right. \\
& \text { or }\left\{\begin{array}{l}
B(X, Y)>0 \\
A_{\mu}(X, Y) C_{\mu}(X, Y)<0 \\
A_{\mu}(X, Y)^{2}-B(X, Y) C_{\mu}(X, Y)^{2}=0
\end{array}\right.
\end{aligned}
$$

We have plotted on Figures 3 and 4 the curves $\left\{A_{\mu}=0\right\},\{B=0\}$ and $\left\{C_{\mu}=0\right\}$ along with the parts of the $(X, Y)$ plane where we have equivalence between the relations $F_{\mu}=0$ and $G_{\mu}=0$. Finally, let us note that $G_{\mu}$ is analytic in the whole plane $(X, Y)$.

\subsection{The case $\mu<1 / 2$}

In this case, we are interested in the points $(0,1 / c)$, for which $B$ vanishes. Consequently, $G_{\mu}$ vanishes at $(0,1 / c)$ if, and only if $F_{\mu}$ vanishes and if, and only if, $A_{\mu}$ vanishes. We have:

$$
A_{\mu}(0,1 / c)=\frac{2 c^{2}\left(\cosh \left(\frac{1}{c}\right)-1\right)}{\mu}-2 c \sinh \left(\frac{1}{c}\right)
$$


The function $\chi: c \mapsto c(\cosh (1 / c)-1) / \sinh (1 / c)$ is strictly increasing, tends to 0 when $c$ tends to 0 and tends to $1 / 2$ when $c$ tends to $+\infty$. Then for all $\mu<1 / 2$, there exists a unique $c(\mu)>0$, such that $A_{\mu}(0,1 / c)=0$. We remark that we have the following property:

$$
\begin{aligned}
\partial G_{\mu}(X, Y)= & 2 A(X, Y) \partial A(X, Y)-\partial B(X, Y) C(X, Y)^{2} \\
& -2 B(X, Y) C(X, Y) \partial C(X, Y) .
\end{aligned}
$$

where $\partial$ denotes a derivative with respect to either $X$ or $Y$

Now $B(X, Y)=Y^{2} X^{2}+4 X$ so we have $\partial_{X} B(0,1 / c(\mu))=4$. Consequently, $\partial_{X} G(0,1 / c(\mu))$ is equal to $-4 C(0,1 / c(\mu))^{2}$ which is not zero (on the contrary, $\partial_{Y} G(0,1 / c(\mu))$ is clearly zero) because we can check that $C_{\mu}(0,1 / c(\mu))=$ $c^{2} \sinh (1 / c)+c>0$. We then have $\psi$ a smooth function (at least $\mathcal{C}^{\infty}$ ) such that $\psi(1 / c(\mu))=0$ and $G_{\mu}(\psi(Y), Y)=0$ for $Y$ in a neighbourhood of $1 / c(\mu)$. Moreover we have $\partial_{Y} \psi(1 / c(\mu))=0$ because $\partial_{Y} G(0,1 / c(\mu))=0$.

We now have to check if the part $Y \neq 0$ of the curve $Y \mapsto(\psi(Y), Y)$, is really the curve $F_{\mu}(X, Y)=0$ so we have to check the signs of $A_{\mu}(\psi(Y), Y)$, $B(\psi(Y), Y)$ and $C_{\mu}(\psi(Y), Y)$ and verify if we are in one of the cases of Equivalence (21). We show that we are in the third case.

We said that we have $C_{\mu}(0,1 / c(\mu))>0$. Then by continuity, for $Y$ not too far from $1 / c(\mu), C_{\mu}(\psi(Y), Y)$ is non negative. We now check the sign of $Y \mapsto B(\psi(Y), Y)$. At $1 / c(\mu)$ this function is zero and one can check that its first derivative also vanishes. Its second derivative however is $4 \psi^{\prime \prime}(1 / c(\mu))=$ $\partial_{Y} A_{\mu}(0,1 / c(\mu))^{2} /\left(2 C_{\mu}(0,1 / c(\mu))^{2}\right)$. On the other hand $Y \mapsto A_{\mu}(\psi(Y), Y)$ vanishes at $1 / c(\mu)$ and its first derivative is $\partial_{Y} A_{\mu}(0,1 / c(\mu))$. We are then going to show that $\partial_{Y} A_{\mu}(0,1 / c(\mu))<0$, which will allow us to conclude that for $Y>1 / c(\mu), A_{\mu}(\psi(Y), Y)<0, C_{\mu}(\psi(Y), Y)>0$ and $B(\psi(Y), Y)>0$ and that $F_{\mu}(\psi(Y), Y)=0$. We note $c$ instead of $c(\mu)$ for the sake of simplicity. We now compute $\partial_{Y} A_{\mu}(0,1 / c(\mu))$ :

$$
\begin{aligned}
\left.\partial_{Y} A_{\mu}(0,1 / c)\right)= & \frac{2}{\mu}\left(c^{2} \sinh (1 / c)-2 c^{3}(\cosh (1 / c)-1)\right) \\
& -2\left(c \cosh (1 / c)-c^{2} \sinh (1 / c)\right) \\
= & \frac{2 c^{2}}{\mu} \sinh (1 / c)-2 c \cosh (1 / c)-\frac{2 c^{3}}{\mu}(\cosh (1 / c)-1)
\end{aligned}
$$

because $\left(2 c^{3}(\cosh (1 / c)-1)\right) / \mu-2 c^{2} \sinh (1 / c)=0$ by definition of $c(\mu)$. Let us now consider the function

$$
\alpha: t \mapsto \frac{2 t^{2} \sinh (1 / t)^{2}}{t(\cosh (1 / t)-1)}-2 t \cosh (1 / t)
$$

we have that $\alpha(c(\mu))-\frac{2 c^{3}}{\mu}(\cosh (1 / c)-1)=\partial_{Y} A_{\mu}(0,1 / c)$ and that $\alpha(t)=-2 t$. We can thus conclude that $\partial_{Y} A_{\mu}(0,1 / c)<0$.

The relation $F_{\mu}(\psi(Y), Y)=0$ leads to $f_{\mu}(\psi(Y), Y \psi(Y))=0$ and the uniqueness property we have for $\phi_{\mu}$ allows us to write $\psi(Y)=\phi_{\mu}(Y \psi(Y))$, for all $Y>1 / c(\mu)$. By letting $Y$ going to $1 / c(\mu)$ we see that we must have $\phi_{\mu}(y) \rightarrow 0$ as $y \rightarrow 0$. In the same manner we have $\frac{\phi_{\mu}(Y \psi(Y))}{Y \psi(Y)}=\frac{1}{Y}$ from which we can conclude that $\phi_{\mu}(y) / y \rightarrow c(\mu)$. We have then shown that $\phi_{\mu}(y) \sim c(\mu) y$ 


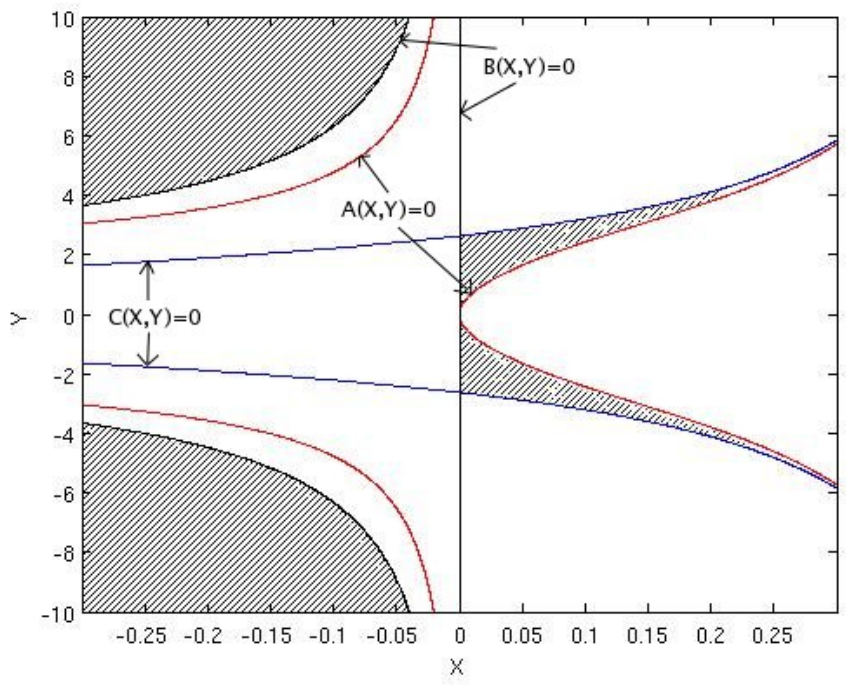

Figure 4: Setting of the $A, B$ and $C$ functions in the $(X, Y)$ plane for $\mu=0.5$. The curve $G_{1 / 2}(X, Y)=0$ needs to start at $(0,0)$ and we see that the behaviour of $A$ and $C$ at this point impose a stronger order of annulation for $\psi$

in a neighbourhood of $y=0$ for $\mu<1 / 2$. In this case we pose $\phi_{\mu}^{\text {app }}(y)=$ $c(\mu) y$. As in the case $\mu>1 / 2$ we look for more information on the expansion of $\phi_{\mu}(y)$. We use the relation $\phi_{\mu}(y)=\psi\left(y / \phi_{\mu}(y)\right)$ and use that $\psi(Y)=1 / 2 \psi^{\prime \prime}(1 / c)(Y-1 / c)^{2}+o(Y-1 / c)^{2}$. We can conclude after some computation that

$$
\left|\phi_{\mu}(y)-\phi_{\mu}^{\mathrm{app}}(y)\right| \sim \sqrt{c^{5} / \psi^{\prime \prime}(1 / c)} y^{3 / 2}
$$

as $y$ goes to 0 . Interestingly enough, we do not have a second term of the expansion since we do not know the sign of the difference.

\subsection{The case $\mu=1 / 2$}

We can treat the case $\mu=1 / 2$ in the same way that we did for $\mu<1 / 2$ by studying the function $G_{1 / 2}$. Firstly we notice that $G_{1 / 2}(0,0)=0$. What's more we have $\partial_{X} G_{1 / 2}(0,0)=-16$ which allows us to use the implicit function theorem (only in this case it is simpler since we do not have constants that depend on $\mu$ ). We still note $\psi$ the function depending on $Y$ such that $\psi(0)=0$ and we have in the vicinity of $(0,0), G_{1 / 2}(X, Y)=0 \Leftrightarrow X=\psi(Y)$. We now check by implicit differentiation that the first three derivative of $\psi$ at 0 vanish and that $\psi^{(4)}(0)=1 / 24$. Consequently $\psi$ is positive in a neighbourhood of $Y=0$. We have $C_{1 / 2}(0,0)=2$ which gives us the sign of $C_{1 / 2}(\psi(Y), Y)$ for small $Y$ and we next have to check the signs of $B(\psi(Y), Y)$ and $A(\psi(Y), Y)$. We check that $Y \mapsto B(\psi(Y), Y)$ has its derivatives at $Y=0$ equal to zero up to order 3 included and that its fourth derivative is non negative at $Y=0$. Thus $B(\psi(Y), Y)$ is non negative for small $Y$. On the other hand, the first derivative of $Y \mapsto A_{1 / 2}(\psi(Y), Y)$ vanishes at $Y=0$ and its second derivative is $-1 / 3$. We 
thus have that $A_{1 / 2}(\psi(Y), Y)$ is negative for small $Y$. We can now conclude that $F_{1 / 2}(\psi(Y), Y)=0$ for $Y$ in a neighbourhood of 0 .

We have again that, $\phi_{1 / 2}(Y \psi(Y))=\psi(Y)$ for all $Y$ small enough. We conclude in the same manner that $\phi_{1 / 2}(y)$ tends to 0 at $y=0$. Moreover, since we have $\psi(Y) \sim c_{0} Y^{4}$ with $c_{0}=1 / 24^{2}$, we have that $\phi_{1 / 2}(y) / y^{4 / 5}$ tends to $c(1 / 2)=c_{0}^{1 / 5}$ when $y$ decreases to 0 . We pose $\phi_{1 / 2}^{\text {app }}(y)=c(1 / 2) y^{4 / 5}$

We now check that $\psi^{(5)}(0)=0$ and $\psi^{(6)}(0) \neq 0$ from what we can conclude that

$$
\phi_{1 / 2}(y)-\phi_{1 / 2}^{\mathrm{app}}(y) \sim c_{1} y^{6 / 5}
$$

\section{Transition on $\tau$ the Macroscopic Stress}

Our aim is to show that there is a transition at $\mu=1 / 2$ for the total stress. The path is now straightforward: we first compute the total stress by integrating $p$ against $\sigma$ then we try to find the behaviour of the total stress as a function of $y$ when $y$ decreases to 0 using our previous results. However some care should be taken as to why the behaviour is correct.

\subsection{The stress $\tau$ as a function of $x$ and $y$}

We first note that if we multiply (1) by $\sigma$ and integrate over the real line we find that, $\int_{|\sigma|>1} \sigma p(\sigma) \mathrm{d} \sigma=\lambda b / D e=y$ (this can be checked by direct computation on the form of $p$ given by (8) but it is longer). We only have to compute $\int_{|\sigma| \leq 1} \sigma p(\sigma) \mathrm{d} \sigma$ and thanks to (8) we find that:

$$
\begin{aligned}
\tau= & \frac{\lambda b}{D e}+\frac{1}{2}\left(-\alpha_{+}\left(1-\rho \beta_{+}\right)+\alpha_{-}\left(1-\rho \beta_{-}\right)\right) \\
& +\rho^{2} \alpha_{+} \beta_{+}\left(1-\rho\left(e^{1 / \rho}-1\right)\right)+\rho^{2} \alpha_{-} \beta_{-}\left(1-\rho\left(1-e^{-1 / \rho}\right)\right) \\
= & I_{1}+I_{2}+I_{3}+I_{4}
\end{aligned}
$$

This formula can be simplified using the definition of $\rho, \beta_{+}$and $\beta_{-}$and the system (12) defining $\alpha_{+}$and $\alpha_{-}$:

$$
\begin{array}{rlrl}
2 I_{2} & =-\alpha_{+}\left(1-\rho \beta_{+}\right)+\alpha_{-}\left(1-\rho \beta_{-}\right) & & \\
& =\rho\left(-\alpha_{+} \beta_{-}+\alpha_{-} \beta_{+}\right) & & \text {using (11) } \\
& =\rho \beta_{+} \beta_{-}\left(\frac{\alpha_{-}}{\beta_{-}}-\frac{\alpha_{+}}{\beta_{+}}\right) & & \\
& =\rho \frac{1}{\mu(-\Gamma)}(-\Gamma) & & \\
& =\frac{\rho}{\mu} &
\end{array}
$$


and

$$
\begin{aligned}
I_{3}+I_{4}= & \rho^{2} \alpha_{+} \beta_{+}\left(1-\rho\left(e^{1 / \rho}-1\right)\right)+\rho^{2} \alpha_{-} \beta_{-}\left(1-\rho\left(1-e^{-1 / \rho}\right)\right) \\
= & \rho^{2}\left(\alpha_{+} \beta_{+}+\alpha_{-} \beta_{-}\right)+\rho^{3}\left(\alpha_{+} \beta_{+}-\alpha_{-} \beta_{-}\right) \\
& -\rho^{3}\left(\alpha_{+} \beta_{+} e^{1 / \rho}-\alpha_{-} \beta_{-} e^{-1 / \rho}\right) \\
= & J_{1}+J_{2}+J_{3}
\end{aligned}
$$

Using (16) we have $\alpha_{+} \beta_{+} e^{1 / \rho}-\alpha_{-} \beta_{-} e^{-1 / \rho}=1 / \mu$ and thus

$$
J_{3}=-\rho^{3} / \mu
$$

For $J_{1}$ and $J_{2}$ we use the values of $\alpha_{+}$and $\alpha_{-}$given by (14). We thus multiply $J_{1}$ by $\Delta$ (given by (13)) to obtain:

$$
\begin{aligned}
\frac{\Delta J_{1}}{\rho^{2}} & =\left(\Delta \alpha_{+}\right) \beta_{+}+\left(\Delta \alpha_{-}\right) \beta_{-} \\
& =\left(\rho \beta_{+}+\rho \beta_{-} e^{-1 / \rho}\right) \beta_{+}+\left(\rho \beta_{-}+\rho \beta_{+} e^{1 / \rho}\right) \beta_{-} \\
& =\rho\left(\beta_{+}^{2}+\beta_{-}^{2}\right)+\rho \beta_{+} \beta_{-}\left(e^{1 / \rho}+e^{-1 / \rho}\right) \\
& =\rho\left(\beta_{+}+\beta_{-}\right)^{2}-2 \rho \beta_{+} \beta_{-}+2 \rho \beta_{+} \beta_{-} \cosh (1 / \rho) \\
& =\frac{1}{\rho}+2 \rho \beta_{+} \beta_{-}(\cosh (1 / \rho)-1)
\end{aligned}
$$

because by defintion of $\beta_{ \pm}$and $\rho$, we have $\beta_{+}+\beta_{-}=1 / \rho$. Now we turn to $J_{2}$

$$
\begin{aligned}
\frac{\Delta J_{2}}{\rho^{3}} & =\left(\Delta \alpha_{+}\right) \beta_{+}-\left(\Delta \alpha_{-}\right) \beta_{-} \\
& =\left(\rho \beta_{+}+\rho \beta_{-} e^{-1 / \rho}\right) \beta_{+}-\left(\rho \beta_{-}+\rho \beta_{+} e^{1 / \rho}\right) \beta_{-} \\
& =\rho\left(\beta_{+}^{2}-\beta_{-}^{2}\right)-\rho \beta_{+} \beta_{-}\left(e^{1 / \rho}-e^{-1 / \rho}\right) \\
& =\beta_{+}-\beta_{-}-2 \rho \beta_{+} \beta_{-} \sinh (1 / \rho)
\end{aligned}
$$

Let us emphasize that we use Cramer's rule and equation (14). That means that $\Delta$ in these expressions is the determinant of system (12) and given by equation (13). In the end, using (24) to (29) we have:

$$
\begin{aligned}
\tau= & \frac{\lambda b}{D e}+\frac{\rho}{2 \mu}-\frac{\rho^{3}}{\mu} \\
& +\frac{\rho^{2}}{\Delta}\left(\frac{1}{\rho}+2 \rho \beta_{+} \beta_{-}(\cosh (1 / \rho)-1)\right) \\
& +\frac{\rho^{3}}{\Delta}\left(\beta_{+}-\beta_{-}-2 \rho \beta_{+} \beta_{-} \sinh (1 / \rho)\right)
\end{aligned}
$$

Recall that we defined in Section 4.3 the variables $x=\mu \Gamma=$ and $y=\lambda b / D e$ and that by (10) we have $\beta_{+} \beta_{-}=-1 / x$. Also we have $\rho=x / y$. We now rewrite 
this equality in the $(x, y)$ variables.

$$
\begin{aligned}
\tau(x, y)= & y+\frac{x}{2 \mu y}-\frac{x^{3}}{\mu y^{3}} \\
& +\frac{x^{2}}{y^{3} \Delta(x, y)}\left(\frac{y^{2}}{x}-2\left(\cosh \left(\frac{y}{x}\right)-1\right)\right) \\
& +\frac{x^{3}}{y^{4} \Delta(x, y)}\left(y\left(\left(\frac{y}{x}\right)^{2}+\frac{4}{x}\right)^{1 / 2}+2 \sinh \left(\frac{y}{x}\right)\right)
\end{aligned}
$$

\subsection{Asymptotical behaviour of $\tau(\phi(y), y)$}

From the previous paragraph we are now able to compute the macroscopic stress $\tau$ attached to a given shear rate $y$. Theoretically, we just have to compute the corresponding $x$ by $x=\phi_{\mu}(y)$ and then to compute the value of $\tau\left(\phi_{\mu}(y), y\right)$ using (31). Now what we are really interested in is the behaviour of the one variable function $y \mapsto \tau\left(\phi_{\mu}(y), y\right)$ at $y=0$. Yet we do not know $\phi_{\mu}$ since it is only given implicitly by (18). However we do know the behaviour of $\phi_{\mu}$ at $y=0$ given by the explicit function $\phi_{\mu}^{\text {app }}$ that we computed in Section 5 . Our hope is to draw from the behaviour of $\tau\left(\phi_{\mu}^{\mathrm{app}}(y), y\right)$ at $y=0$, the behaviour of $\tau\left(\phi_{\mu}(y), y\right)$. By behaviour we mean that we want to find the first non zero term of the expansion of $\tau\left(\phi_{\mu}(y), y\right)$.

Theoretical inequality. We first look at the kind of inequality we are going to use. Let us forget for a moment the origin of our problem and say we are given a smooth two variable function $\widetilde{\tau}$ that we want to study alongside a curve given by $(\phi(y), y)$. Assume $\phi^{\mathrm{app}}(y)$ is equivalent to $\phi(y)$. By using Taylor's formula with integral remainder on $\widetilde{\tau}$ we get the following inequality.

$$
\begin{aligned}
\left|\frac{\widetilde{\tau}(\phi(y), y)}{\widetilde{\tau}\left(\phi^{\mathrm{app}}(y), y\right)}-1\right| & =\left|\frac{\widetilde{\tau}(\phi(y), y)-\widetilde{\tau}\left(\phi^{\mathrm{app}}(y), y\right)}{\widetilde{\tau}\left(\phi^{\mathrm{app}}(y), y\right)}\right| \\
& \leq\left|\frac{\phi(y)-\phi^{\mathrm{app}}(y)}{\widetilde{\tau}\left(\phi^{\mathrm{app}}(y), y\right)}\right| \int_{0}^{1}\left|\partial_{x} \widetilde{\tau}\left(s \phi(y)+(1-s) \phi^{\mathrm{app}}(y), y\right)\right| \mathrm{d} s
\end{aligned}
$$

When we see this inequality we see that we need the behaviour of $\left|\phi(y)-\phi^{\mathrm{app}}(y)\right|$. Actually we need it for two reasons. Firstly we need the precise behaviour of $\left|\frac{\phi(y)-\phi^{\text {app }}(y)}{\widetilde{\tau}\left(\phi^{\operatorname{app}}(y), y\right)}\right|$. Secondly if we hope to control the integral we need to have an estimation of the position of the segment $\left[(\phi(y), y),\left(\phi^{\operatorname{app}}(y), y\right)\right]$ in the $(x, y)$ plane which is given by a second term in the expansion of $\phi$.

What we wanted to point out by this little study is that there is absolutely no guarantee that the behaviour of $\widetilde{\tau}(\phi(y), y)$ is given by $\widetilde{\tau}\left(\phi^{\mathrm{app}}(y), y\right)$ beforehand. We have to prove that the right hand side of (32) tends to 0 which is not obvious at all and can be false even if $\phi(y) \sim \phi^{\mathrm{app}}(y)$.

The problem with $\tau$ given by (31) and the introduction of $\widetilde{\tau}$. We now want to apply (32) to $\tau$ given by (31). As a matter of fact if we use (17) and (31) on the curve $\left(\phi_{\mu}^{\text {app }}(y), y\right)$ as we planned, we fail to recover the correct behaviour for $\tau\left(\phi_{\mu}(y), y\right)$ except for $\mu<1 / 2$. We can in fact compute that in case $\mu=1 / 2$, 
$\tau\left(\phi_{\mu}^{\text {app }}(y), y\right)$ follows a power law with exponent $1 / 5$ but with a different constant than the one given by Theorem 1 while in the case $\mu>1 / 2, \tau\left(\phi_{\mu}^{\operatorname{app}}(y), y\right)$ diverges as $1 / y$ when it should converge as $y$ (again according to Theorem 1 written in $(x, y)$ variables). This leaves us with the question of how to prove the theorem.

Remember that in the end what we are really interested in is the behaviour of $\tau\left(\phi_{\mu}(y), y\right)$ not $\tau\left(\phi_{\mu}^{\mathrm{app}}(y), y\right)$. We now use the implicit definition of $\phi_{\mu}$ to construct a two variables function $\widetilde{\tau}$ such that $\tau\left(\phi_{\mu}(y), y\right)=\widetilde{\tau}\left(\phi_{\mu}(y), y\right)$ and that $\widetilde{\tau}\left(\phi_{\mu}^{\text {app }}(y), y\right)$ gives the correct behaviour for $\phi_{\mu}$ that is to say that we can prove that the right hand side of $(32)$ tends to 0 .

We make the following remark: the equation (18) which defines implicitly $\phi_{\mu}$ can be put in an equivalent form $\Delta(x, y)=\widetilde{\Delta}(\mu, x, y)$ with

$$
\widetilde{\Delta}(\mu, x, y)=\mu\left(\sqrt{\frac{y^{2}}{x^{2}}+\frac{4}{x}} \cosh \left(\frac{y}{x}\right)+\left(\frac{2}{y}+\frac{y}{x}\right) \sinh \left(\frac{y}{x}\right)\right)
$$

and $\Delta$ is given by equation (17) and is the quantity that appears in the expression of $\tau(x, y)$ given by $(31)$. This is in fact how we found the function $f_{\mu}$ in the first place in section 4.3 Consequently if we define $\widetilde{\tau}$ by

$$
\begin{aligned}
\widetilde{\tau}(x, y)= & y+\frac{x}{2 \mu y}-\frac{x^{3}}{\mu y^{3}} \\
& +\frac{x^{2}}{y^{3} \widetilde{\Delta}(\mu, x, y)}\left(\frac{y^{2}}{x}-2\left(\cosh \left(\frac{y}{x}\right)-1\right)\right) \\
& +\frac{x^{3}}{y^{4} \widetilde{\Delta}(\mu, x, y)}\left(y\left(\left(\frac{y}{x}\right)^{2}+\frac{4}{x}\right)^{1 / 2}+2 \sinh \left(\frac{y}{x}\right)\right)
\end{aligned}
$$

we know that we have $\tau\left(\phi_{\mu}(y), y\right)=\widetilde{\tau}\left(\phi_{\mu}(y), y\right)$ and we will show that $\widetilde{\tau}$ is the correct function to consider to study (32). Now this raises the question as to why $\widetilde{\tau}$ works and not $\tau$ and whether this fact was predictible.

Behaviour of $\left|\frac{\phi(y)-\phi^{\mathrm{app}}(y)}{\widetilde{\tau}\left(\phi^{\mathrm{app}}(y), y\right)}\right|$. To control the right hand side of (32) we see that we need the behaviour of $\widetilde{\tau}_{\mu}\left(\phi^{\mathrm{app}}(y), y\right)$ which we give now:

If $\mu>1 / 2, \tilde{\tau}(\phi(y), y) \sim \eta(\mu) y$.

If $\mu<1 / 2, \tilde{\tau}(\phi(y), y) \rightarrow \tau_{0}(\mu)$.

If $\mu=1 / 2, \widetilde{\tau}(\phi(y), y) \sim 24^{2 / 5} / 12 y^{1 / 5}$.

In the end these behaviours will also be the respective behaviours of $\tau\left(\phi_{\mu}(y), y\right)$. Using this information and the one from $\phi_{\mu}-\phi_{\mu}^{\text {app }}$ given by equations (20), (22) and (23) we can say that

Proposition 4. We have the following behaviours :

If $\mu>1 / 2,\left|\frac{\phi(y)-\phi^{\mathrm{app}}(y)}{\widetilde{\tau}\left(\phi^{\mathrm{app}}(y), y\right)}\right|$ behaves like $y$.
If $\mu<1 / 2,\left|\frac{\phi(y)-\phi^{\mathrm{app}}(y)}{\widetilde{\tau}\left(\phi^{\mathrm{app}}(y), y\right)}\right|$ behaves like $y^{3 / 2}$. 
If $\mu=1 / 2,\left|\frac{\phi(y)-\phi^{\mathrm{app}}(y)}{\widetilde{\tau}\left(\phi^{\mathrm{app}}(y), y\right)}\right|$ also behaves like $y$.

where we have dropped the constants for the sake of clarity. All these computations are straightforward since everything is explicit.

End of the proof: study of $\int_{0}^{1}\left|\partial_{x} \widetilde{\tau}\left(s \phi(y)+(1-s) \phi^{\operatorname{app}}(y), y\right)\right| \mathrm{d} s$

The case $\mu>1 / 2$. As usual, let us start with the simplest case $\mu>1 / 2$. Using Proposition 3 we have that $\phi_{\mu}$ converges to a constant $c>0$. The function $\partial_{x} \widetilde{\tau}$ can be continued by continuity at $(c, 0)$ when $c>0$. It is then bounded in the vicinity of this point. Now we know that for $y$ close enough to 0 , $\left[\left(\phi_{\mu}(y), y\right),\left(\phi_{\mu}^{\mathrm{app}}(y), y\right)\right]$ is in any closed ball centered at $(c, 0)$. By compactness $d_{x} \widetilde{\tau}$ is bounded and thus the integral is uniformly bounded as $y$ decreases to zero and we then have $\tau(\phi(y), y) \sim \widetilde{\tau}\left(\phi^{\text {app }}(y), y\right)$. As a matter of fact if we look at (32) what we have really proved is the following expansion:

$$
\tau\left(\phi_{\mu}(y), y\right)=\eta(\mu) y+\mathcal{O}\left(y^{2}\right)
$$

For the other two cases the difficulty is increased since we have $\phi_{\mu}$ and $\phi_{\mu}^{\text {app }}$ closing in to 0 . But the dependence in $y / x$ of $\widetilde{\tau}_{\mu}$ and thus of $\partial_{x} \widetilde{\tau}$ leave us no hope to ever show continuity at $(0,0)$ and uniform boundedness of the integral. In fact we can see numerically that the function diverges. What we have to prove is that the divergence of the integral is somehow compensated by $\left|\frac{\phi(y)-\phi^{\operatorname{app}}(y)}{\widetilde{\tau}\left(\phi^{\operatorname{app}}(y), y\right)}\right|$.

The case $\mu<1 / 2$. In the $\mu<1 / 2$ case we know by Proposition 3 that for $y$ close to 0 , the curve $\left\{\left(\phi_{\mu}(y), y\right)\right\}$ is tangent to a ray $\{(c(\mu) y, y)\}$. Now we look at the expansion of $\partial_{x} \widetilde{\tau}(x, y)$ alongside a ray $(y / t, y)$ and we find that its first term is of the form $M(t) / y$ with $M(t)$ bounded if $t$ is in $\mathbf{R}$. This suggests that the divergence of $\partial_{x} \widetilde{\tau}(x, y)$ is uniformly in $1 / y$ close to a ray. Indeed, if we check the function $(t, y) \mapsto y \partial_{x} \widetilde{\tau}(y / t, y)$ we can see that it can be continuously continued up to $y=0$ as long as $t$ is non negative. This means that as long as $(x, y)$ is in a closed angular sector with its peak at $(0,0)$ and included in the $x>0, y>0$ open quarter plane (which means that none of its edges are axis), $y \partial_{x} \widetilde{\tau}(x, y)$ is bounded.

Thus, we just have to check that as $y$ decreases to 0 , the segment $\left[\left(\phi_{\mu}(y), y\right)\right.$, $\left.\left(\phi_{\mu}^{\mathrm{app}}(y), y\right)\right]$ stays in such a closed angular sector, so as to have $\int_{0}^{1} \mid \partial_{x} \widetilde{\tau}\left(s \phi_{\mu}(y)+\right.$ $\left.(1-s) \phi_{\mu}^{\mathrm{app}}(y), y\right) \mid \mathrm{d} s \leq M / y$ for a given constant $M$ which does not depend on $y$ (but can depend on $\mu$ ). This is true because of Proposition 3 which states that $\phi_{\mu}(y) / y \rightarrow c>0 \mid$. .

Since by Proposition $4\left|\frac{\phi_{\mu}(y)-\phi_{\mu}^{\mathrm{app}}(y)}{\widetilde{\tau}\left(\phi_{\mu}^{\mathrm{app}}(y), y\right)}\right|$ is in this case of order $y^{3 / 2}$, what we just show is enough to conclude that $\tau\left(\phi_{\mu}(y), y\right) \sim \widetilde{\tau}\left(\phi_{\mu}^{\operatorname{app}}(y), y\right)$. Again we have proved slightly more than announced since we have the expansion

$$
\tau\left(\phi_{\mu}(y), y\right)=\tau_{0}(\mu)+\mathcal{O}(\sqrt{y})
$$

It would actually be interesting to now if $\tau\left(\phi_{\mu}(y), y\right)-\tau_{0}(\mu) \sim \sqrt{y}$ to know what Herschell-Bulkley fluid law best fits the behaviour of a HL fluid at low shear rate. 
The case $\mu=1 / 2$. What has been said should make us worry over the $\mu=1 / 2$ case. Indeed in this case, by looking back at Proposition 4 , we see that we need a divergence of the integral that is strictly weaker that $1 / y$ and it seems that we have shown that the divergence is of order $1 / y$ when closing in to $(0,0)$. In reality, one should notice that $M(t)$ tends to 0 as $t$ tends to 0 , that is to say when the half-line we are considering becomes close to the $(O x)$ axis. In the $\mu=1 / 2$ case, $\phi_{1 / 2}^{\text {app }}(y)=1 / 24^{2 / 5} y^{4 / 5}$ which means that the $(O x)$ axis is tangent to the curves. The previous divergence rate does not apply here. In fact we have $\left.\partial_{x} \widetilde{\tau}\left(t y^{4 / 5}, y\right)\right)$ uniformly bounded by $M / y^{3 / 5}$ as long as $t$ is away from 0 which is the case in the region where $\left[(\phi(y), y),\left(\phi^{\operatorname{app}}(y), y\right)\right]$ lies.

We will not write the full expression of $\partial_{x} \widetilde{\tau}\left(t y^{4 / 5}, y\right)$ but we will say that it can be written as the sum of 8 terms and all of them are diverging faster than $y^{3 / 5}$. But all these terms can in fact be taken by pairs so as to alleviate the strong divergence. For example we have the term $1 / y$ and the term:

$$
\begin{aligned}
& T=-\frac{t^{2}\left(\frac{y^{6 / 5}}{t}-2 \cosh \left(\frac{y^{1 / 5}}{t}\right)+2\right)}{y^{7 / 5}\left(\frac{1}{2} \sqrt{\frac{y^{2 / 5}}{t^{2}}+\frac{4}{t y^{4 / 5}}} \cosh \left(\frac{y^{1 / 5}}{t}\right)+\left(\frac{1}{y}+\frac{y^{1 / 5}}{2 t}\right) \sinh \left(\frac{y^{1 / 5}}{t}\right)\right)^{2}} \times \\
& {\left[\frac{1}{4} \frac{\cosh \left(\frac{y^{1 / 5}}{t}\right)}{\sqrt{\frac{y^{2 / 5}}{t^{2}}+\frac{4}{t y^{4 / 5}}}}\left(-\frac{2}{y^{2 / 5} t^{3}}-\frac{4}{t^{2} y^{8 / 5}}\right)-\frac{1}{2} \sqrt{\frac{y^{2 / 5}}{t^{2}}+\frac{4}{t y^{4 / 5}} \frac{\sinh \left(\frac{y^{1 / 5}}{t}\right)}{y^{3 / 5} t^{2}}}\right.} \\
& \left.-\frac{1}{2} \frac{\sinh \left(\frac{y^{1 / 5}}{t}\right)}{y^{3 / 5} t^{2}}-\left(\frac{1}{y}+\frac{y^{1 / 5}}{2 t}\right) \frac{\cosh \left(\frac{y^{1 / 5}}{t}\right)}{y^{3 / 5} t^{2}}\right]
\end{aligned}
$$

which happens to be equivalent to $-1 / y$ as $y$ tends to 0 with $t$ fixed. The sum of these two terms is also equivalent to $1 / y^{3 / 5}$. As in the previous paragraph this is not enough since we want some uniformity in $t$ but it suggests that by adding and then multiplying by $y^{3 / 5}$ we might get what we are looking for. We first rewrite (34) in order to suppress any obvious singularities: 


$$
\begin{gathered}
T=-\frac{t^{2}\left(\frac{y^{4 / 5}}{t}-\frac{2 \cosh \left(\frac{y^{1 / 5}}{t}\right)-2}{y^{2 / 5}}\right)}{\left(\frac{1}{2} y^{2 / 5} \sqrt{\frac{y^{6 / 5}}{t^{2}}+\frac{4}{t}} \cosh \left(\frac{y^{1 / 5}}{t}\right)+\left(\frac{1}{y^{1 / 5}}+\frac{y}{2 t}\right) \sinh \left(\frac{y^{1 / 5}}{t}\right)\right)^{2}} \times \\
{\left[\frac{1}{4} \frac{\cosh \left(\frac{y^{1 / 5}}{t}\right)}{\sqrt{\frac{y^{6 / 5}}{t^{2}}+\frac{4}{t}}}\left(-\frac{2 y^{3 / 5}}{t^{3}}-\frac{4}{t^{2} y^{3 / 5}}\right)-\frac{1}{2} \sqrt{\frac{y^{6 / 5}}{t^{2}}+\frac{4}{t} \frac{\sinh \left(\frac{y^{1 / 5}}{t}\right)}{y^{2 / 5} t^{2}}}\right.} \\
\left.-\frac{1}{2} \frac{\sinh \left(\frac{y^{1 / 5}}{t}\right)}{t^{2}}-\left(\frac{1}{y}+\frac{y^{1 / 5}}{2 t}\right) \frac{\cosh \left(\frac{y^{1 / 5}}{t}\right)}{t^{2}}\right]
\end{gathered}
$$

Now the denominator, which we note by

$$
\mathcal{D}(t, y)=\left(\frac{1}{2} y^{2 / 5} \sqrt{\frac{y^{6 / 5}}{t^{2}}+\frac{4}{t}} \cosh \left(\frac{y^{1 / 5}}{t}\right)+\left(\frac{1}{y^{1 / 5}}+\frac{y}{2 t}\right) \sinh \left(\frac{y^{1 / 5}}{t}\right)\right)^{2},
$$

is never 0 since $\sinh (u) / u$ is bounded away from 0 and so is $t$. We now see that the part that is dominant in the numerator of $T$ when taking $y$ to 0 with $t$ fixed is $\cosh \left(y^{1 / 5} / t\right) / y$. We can then rewrite $T$ as

$$
T=\frac{S\left(t, y^{1 / 5}\right)+\frac{t^{2}\left(\frac{y^{4 / 5}}{t}-\frac{2 \cosh \left(\frac{y^{1 / 5}}{t}\right)-2}{y^{2 / 5}}\right) \cosh \left(\frac{y^{1 / 5}}{t}\right)}{y t^{2}}}{\mathcal{D}\left(t, y^{1 / 5}\right)}
$$

where $S$ contains all the terms in the numerator of $T$ of lower order (again $y$ tends to 0 and $t$ is fixed). It is easy to check that in fact $u^{3} S(t, u)$ is a continuous function of its arguments as long as $t$ is bounded away from 0 . In particular we have that in any rectangle $[a, b] \times[0, \varepsilon]$ for which $b>a>0 u^{3} S(t, u)$ is bounded which means that $y^{3 / 5} S\left(t, y^{1 / 5}\right)$ is bounded.

Now for the dominant part we need to reduce to a common denominator. We expand the denominator:

$$
\mathcal{D}\left(t, y^{1 / 5}\right)=\widehat{\mathcal{D}}\left(t, y^{1 / 5}\right)+\left(\frac{\sinh \left(\frac{y^{1 / 5}}{t}\right)}{y^{1 / 5}}\right)^{2}
$$


so that in $\widehat{\mathcal{D}}(t, u)$ we have collected all the terms that tend to 0 as $u$ tends to 0 . In fact we can even check that $\widehat{\mathcal{D}}(t, u) / u^{2}$ is a countinuous function. Now we compute $y^{3 / 5}(1 / y+T)$ using (37) and (38) to see that we have:

$$
\begin{aligned}
& y^{3 / 5}(1 / y+T)=\frac{y^{3 / 5}}{\mathcal{D}\left(t, y^{1 / 5}\right)}\left[\frac{\widehat{\mathcal{D}}\left(t, y^{1 / 5}\right)}{y}+S\left(t, y^{1 / 5}\right)\right. \\
& \left.+\left(\frac{1}{y}\left(\frac{\sinh \left(\frac{y^{1 / 5}}{t}\right)}{y^{1 / 5}}\right)^{2}-\frac{1}{y}\left(\frac{2 \cosh \left(\frac{y^{1 / 5}}{t}\right)-2}{y^{2 / 5}}\right) \cosh \left(\frac{y^{1 / 5}}{t}\right)\right)\right]
\end{aligned}
$$

In this expression we have seen that all the terms have been accounted for except for the last one, which was the most problematic. One can check that in fact this last term can be seen as a continuous function of $y^{1 / 5} / t$ (it can even be expanded in a power series of $y^{1 / 5} / t$ ) and thus is also bounded as long as $t$ stays away from 0 . The rest of $y^{3 / 5} \partial_{x} \widetilde{\tau}\left(t y^{4 / 5}, y\right)$ can be treated in the same way. and can be considered a bounded function uniformly in $y$ and $t$. Now thanks to the fact that by Proposition 3 for $\mu=1 / 2$ we have $\phi_{1 / 2}^{\text {app }}(y)=1 / 24^{2 / 5} y^{4 / 5}$ and that by (23) $\left|\phi_{1 / 2}(y)-\phi_{1 / 2}^{\mathrm{app}}(y)\right| / y^{4 / 5}$ is bounded as $y$ tends to 0 , we have that $\int_{0}^{1}\left|\partial_{x} \widetilde{\tau}\left(s \phi_{1 / 2}(y)+(1-s) \phi_{1 / 2}^{\text {app }}(y), y\right)\right| \mathrm{d} s \leq M / y^{3 / 5}$ and we can conclude in this case too, using again (32) and (4), that $\tau\left(\phi_{1 / 2}(y), y\right) \sim \widetilde{\tau}\left(\phi_{1 / 2}^{\text {app }}(y), y\right)$ and we even have:

$$
\widetilde{\tau}\left(\phi_{1 / 2}(y), y\right)=c(\mu) y^{1 / 5}+\mathcal{O}\left(y^{2 / 5}\right)
$$

Acknowledgement : The author is supported by the French National Agency Research project MANIPHYC 2009-2012 managed by L. Bocquet. The author would also like to thank D. Bresch, S. Descombes and E. Oudet for their involvement and support.

\section{A Proof of uniqueness in Theorem 1}

We give in this section some details on the computations for the uniqueness part of Theorem 1. Remember that we have:

$$
\begin{array}{r}
g(x, y)=\frac{\frac{2 x^{2}}{y^{2}}\left(\cosh \left(\frac{y}{x}\right)-1\right)+x \cosh \left(\frac{y}{x}\right)+\left(\frac{3 x^{2}}{y}+x y\right) \sinh \left(\frac{y}{x}\right)}{\sqrt{y^{2}+4 x} \cosh \left(\frac{y}{x}\right)+\left(\frac{2 x}{y}+y\right) \sinh \left(\frac{y}{x}\right)} \\
\frac{+\sqrt{y^{2}+4 x}\left(\frac{x^{2}}{y^{2}}\left(\cosh \left(\frac{y}{x}\right)-1\right)+\frac{x}{y} \sinh \left(\frac{y}{x}\right)+x \cosh \left(\frac{y}{x}\right)\right)}{\sqrt{y^{2}+4 x} \cosh \left(\frac{y}{x}\right)+\left(\frac{2 x}{y}+y\right) \sinh \left(\frac{y}{x}\right)}
\end{array}
$$

and we want to prove that $\partial_{x} g(x, y)>0$ for any given $x$ and $y$ both non negative. We first compute $\partial_{x} g(x, y)$ : 


$$
\begin{aligned}
& \partial_{x} g(x, y)=\frac{1}{\sqrt{y^{2}+4 x} \cosh \left(\frac{y}{x}\right)+\left(2 \frac{x}{y}+y\right) \sinh \left(\frac{y}{x}\right)} \times \\
& {\left[\frac{4 x}{y^{2}}\left(\cosh \left(\frac{y}{x}\right)-1\right)-\frac{2}{y} \sinh \left(\frac{y}{x}\right)+\cosh \left(\frac{y}{x}\right)-\frac{y}{x} \sinh \left(\frac{y}{x}\right)+\left(\frac{6 x}{y}+y\right) \sinh \left(\frac{y}{x}\right)\right.} \\
& -\left(3+\frac{y^{2}}{x}\right) \cosh \left(\frac{y}{x}\right)+\frac{2\left(\frac{x^{2}}{y^{2}}\left(\cosh \left(\frac{y}{x}\right)-1\right)+\frac{x}{y} \sinh \left(\frac{y}{x}\right)+x \cosh \left(\frac{y}{x}\right)\right)}{\sqrt{y^{2}+4 x}} \\
& \left.+\sqrt{y^{2}+4 x}\left(\frac{2 x}{y^{2}}\left(\cosh \left(\frac{y}{x}\right)-1\right)-\frac{\cosh \left(\frac{y}{x}\right)}{x}+\cosh \left(\frac{y}{x}\right)-\frac{y}{x} \sinh \left(\frac{y}{x}\right)\right)\right] \\
& \left.-\frac{1}{\left(\sqrt{y^{2}}+4 x\right.} \cosh \left(\frac{y}{x}\right)+\left(2 \frac{x}{y}+y\right) \sinh \left(\frac{y}{x}\right)\right)^{2} \times \\
& {\left[\frac{2 \cosh \left(\frac{y}{x}\right)}{\left.\sqrt{y^{2}+4 x}-\frac{y}{x^{2}} \sqrt{y^{2}+4 x} \sinh \left(\frac{y}{x}\right)+\frac{2}{y} \sinh \left(\frac{y}{x}\right)-\left(\frac{2}{x}+\frac{y^{2}}{x^{2}}\right) \cosh \left(\frac{y}{x}\right)\right] \times}\right.} \\
& {\left[2 \frac{x^{2}}{y^{2}}\left(\cosh \left(\frac{y}{x}\right)-1\right)+x \cosh \left(\frac{y}{x}\right)+\left(3 \frac{x^{2}}{y}+x y\right) \sinh \left(\frac{y}{x}\right)\right.} \\
& \left.+\sqrt{y^{2}+4 x}\left(\frac{x^{2}}{y^{2}}\left(\cosh \left(\frac{y}{x}\right)-1\right)+\frac{x}{y} \sinh \left(\frac{y}{x}\right)+x \cosh \left(\frac{y}{x}\right)\right)\right]
\end{aligned}
$$

To handle such an expression (whose sign is anything but obvious) we reduce to the same denominator and write the numerator and the denominator as polynomials of the variables $x, y, c=\cosh (y / x), s=\sinh (y / x)$, and $r=$ $\sqrt{y^{2}+4 x}$. We obtain:

$$
\begin{aligned}
& \partial_{x} g(x, y)=\frac{1}{y\left(r c y+2 x s+s y^{2}\right)^{2} r} \times \\
& \left(-4 x y^{3}+4 x^{2} y+2 y^{5} c^{2}-12 x^{3} s-2 y^{4} s-y^{5} c-y^{5} r+14 x^{2} c^{2} r y\right. \\
& +4 x^{2} r c s-4 x r c y-8 x y^{2} r s-2 x y^{3} r c-8 x^{2} y r c-y^{5}+8 r x c s y^{2}+2 y^{5} c^{2} r \\
& +2 y^{6} c s+2 c y^{4} r s+12 x c^{2} r y^{3}+34 x^{2} y^{2} c s+16 x y^{4} c s+12 x c^{2} y^{3}+16 x^{2} c^{2} y \\
& \quad-6 x^{2} y r-2 x y^{4} s-8 x y^{2} s-4 x^{2} r s+4 x r y+12 x^{3} c s-2 y^{3} r c-6 x y^{3} r \\
& \left.-12 x^{2} y^{2} s-y^{4} r s-20 x^{2} c y-10 x y^{3} c\right)
\end{aligned}
$$

As $y$ and $x$ are non negative $c, s$ and $r$ are also non negative. So the denominator is non negative and in the numerator, a monomial is non negative if it has a plus and negative if it has a minus. The trick to show that the numerator is non negative is to combine monomial and use the fact that $c-1>0$ and $s-y / x>0$. Let us give an example. The numerator contains $16 x^{2} y^{2} c^{2}+4 x^{2} y+$ $12 x^{3} c s-8 x y^{2}-12 x^{3} s-20 x^{2} y c$ (expression which in facts contains the negative 
monomial that were the most difficult to combine to obtain a non negative quantity). One can check that in fact this expression can be written under the form $8 x^{2} y c(c-1)+12 x^{3}(c-1)(s-y / x)+8 x^{2} y s(s-y / x)$ which is now clearly non negative. As a result we obtain (we write $t$ instead of $y / x$ ):

$$
\begin{aligned}
& \partial_{x} g(x, y)=\frac{1}{y\left(r c y+2 x s+s y^{2}\right)^{2} r} \times \\
& {\left[2 x y^{3} r c(c-1)+12 x^{3}(c-1)(s-t)+2 x y^{3}\left(c^{2}-1\right)+y^{5} r\left(c^{2}-1\right)\right.} \\
& +y^{5} c(c-1)+y^{5}\left(c^{2}-1\right)+2 x y^{2} r c(s-t)+6 x^{2} y r s(s-t)+2 x y^{2} r s(c-1) \\
& +8 x^{2} y r c(c-1)+y^{4} r s(c-1)+4 x^{2} r(c-1)(s-t)+12 x^{2} y^{2} s(c-1) \\
& \quad+2 x y^{3} s(s-t)+8 x^{2} y s(s-t)+2 x^{2} y^{2} c(s-t)+8 x^{2} y c(c-1) \\
& \quad+8 x y^{3} c(c-1)+2 x y^{4} s(c-1)+6 x y^{3} r\left(c^{2}-1\right)+4 r x c s y^{2} \\
& \left.\quad+y^{5} c^{2} r+2 y^{6} c s+c y^{4} r s+4 x c^{2} r y^{3}+20 x^{2} y^{2} c s+14 x y^{4} c s\right]
\end{aligned}
$$

which is now the sum of non negative term, hence the result.

\section{Contents}

1 Introduction

2 Hébraud-Lequeux Model 2

3 Presentation of the Main Results 4

4 Construction of the Solution 6

4.1 Solving the linear equation in $\mathcal{D}^{\prime}\left(\mathbf{R}^{*}\right) \ldots \ldots \ldots \ldots$

4.2 Solving the nonlinear problem $(3)-(4) \ldots \ldots \ldots \ldots \ldots$

4.3 Implicit relations that link $\Gamma$ and $b \ldots \ldots \ldots \ldots$

5 Asymptotic Behaviour of $\phi_{\mu}(y)$ at $y \rightarrow 0 \quad 11$

5.1 The simplest case $\mu>1 / 2 \ldots \ldots \ldots \ldots \ldots \ldots$

5.2 Preliminary computation for cases $\mu \leq 1 / 2 \ldots \ldots \ldots \ldots \ldots$

5.3 The case $\mu<1 / 2 \ldots \ldots \ldots \ldots \ldots \ldots \ldots$

5.4 The case $\mu=1 / 2 \ldots \ldots \ldots \ldots \ldots \ldots \ldots$

6 Transition on $\tau$ the Macroscopic Stress 17

6.1 The stress $\tau$ as a function of $x$ and $y \ldots \ldots \ldots \ldots \ldots$

6.2 Asymptotical behaviour of $\tau(\phi(y), y) \ldots \ldots \ldots \ldots$

A Proof of uniqueness in Theorem 1 


\section{References}

[1] Mohamed Ben Alaya and Benjamin Jourdain. Probabilistic approximation of a nonlinear parabolic equation occurring in rheology. J. Appl. Probab., 44(2):528-546, 2007.

[2] Eric Cancès, Isabelle Catto, and Yousra Gati. Mathematical analysis of a nonlinear parabolic equation arising in the modelling of non-Newtonian flows. SIAM J. Math. Anal., 37(1):60-82 (electronic), 2005.

[3] Eric Cancès, Isabelle Catto, Yousra Gati, and Claude Le Bris. Wellposedness of a multiscale model for concentrated suspensions. Multiscale Model. Simul., 4(4):1041-1058 (electronic), 2005.

[4] Eric Cancès and Claude Le Bris. Convergence to equilibrium of a multiscale model for suspensions. Discrete Contin. Dyn. Syst. Ser. B, 6(3):449-470 (electronic), 2006.

[5] Yousra Gati. Analyse mathématique et simulations numériques d'un modèle de fluides complexes. PhD thesis, École Nationale des Ponts et Chaussées, 2004.

[6] Pascal Hébraud and François Lequeux. Mode-coupling theory for the pasty rheology of soft glassy materials. Phys. Rev. Lett., 81(14):2934-2937, Oct 1998.

[7] Oswald Patrick. Rhéophysique. Belin, 2005. 